\title{
Parámetros limnológicos del área de influencia del cultivo de cachama (Piaractus brachypomus) en jaulas
}

\author{
Limnological parameters in area of influence of cachama \\ (Piaractus brachypomus) cages culture. \\ Parâmetros limnológicos da área de influência do cultivo da \\ pirapitinga (Piaractus brachypomus) em gaiolas
}

Martha Yossa ${ }^{1}$

1 Zootec, MSc, PhD, Grupo de investigación en alimentación y nutrición de organismos acuáticos-GRANAC, Instituto de Acuicultura de los Llanos. Universidad de los Llanos, km 12 vía Puerto López, Villavicencio, Meta-Colombia.

Email: granac.iall@unillanos.edu.co

Recibido: 04 de mayo de 2016

Aceptado: 22 de mayo de 2018

\begin{abstract}
Resumen
Para caracterizar el área de influencia del cultivo de cachama en jaulas sobre la calidad del agua y sedimento en la Ensenada Soplaviento en el río Manacacias, fueron monitoreados: oxígeno, temperatura, pH, alcalinidad, dureza, amonio, ortofosfato, conductividad, sólidos totales, transparencia, profundidad y materia orgánica. El fósforo total y el nitrógeno fueron usados como indicadores para determinar el grado de trófia por Índices de Estado Trófico (IET). Para temperatura, $\mathrm{pH}$, dureza, conductividad y sólidos no fue encontrado efecto significativo del muestreo, lo que sugiere que el muestreo asociado a los ciclos de lluvia influencian de manera acentuada los parámetros de calidad de agua en este tipo de sistemas. En términos de estado trófico las condiciones permanecieron mesotróficas denotándose que el sistema implementado no representa aún saturación para el medio.
\end{abstract}

Palabras clave: eutrofización, materia orgánica, río Manacacias, sedimento.

\begin{abstract}
The parameters of oxygen, temperature, $\mathrm{pH}$, alkalinity, hardness, ammonium, orthophosphate, conductivity, totals solids, transparency, depth and organic matter were monitored to characterize the area of influence of cage culture on water and sediment quality in the Soplaviento Ensenada in the Manacacias River. Trophic State Indexes (IET) estimated phosphorus and nitrogen to determine the trophic state. For temperature, $\mathrm{pH}$, hardness, conductivity and totals solids, not significant effect of sampling was found; suggesting that sampling associated with rainfall cycles strongly influences water quality parameters in this type of system. In terms of trophic status the conditions remained mesotrophic, denoting that the system implemented does not yet represent saturation for the medium.
\end{abstract}

Key Words: eutrophication, Manacacias river, organic matter, sediment 


\begin{abstract}
Resumo
Parâmetros fisicos e quimicos da qualidade da agua e a matéria orgânica no sedimento, foram monitorados para caracterizar a área de influência do cultivo da pirapitinga em gaiola na Ensenada Soplaviento no rio Manacacias. O fósforo total e o nitrogênio foram usados no grau de trofia através do índice de estado trófico (IET). Para temperatura, pH, dureza, condutividade e sólidos totais, nao foi encontrado um efeito significativo da amostragem, o que sugere amostragem associada a ciclos de chuvas influencia fortemente os parâmetros de qualidade da água neste tipo de sistema. Em termos gerais as condições do estado trófico ficaram mesotróficas, indicando que o sistema implementado ainda não representa risco de saturação para o ambiente.
\end{abstract}

Palavras chave: eutrofização, matéria orgânica, rio Manacacias, sedimento.

\section{Introducción}

La piscicultura se destaca como una actividad fundamental para la seguridad alimentaria y la reducción de la pobreza, y el crecimiento global de la actividad es ampliamente registrado por la FAO (2018). En Colombia, durante los últimos años el incremento piscícola aproximado fue de $33 \%$ promedio anual y se desarrolló en estanques excavados en tierra ( $66 \%$ del total de producción) y en jaulas, que con menos del $2 \%$ del espejo de agua aportó el 44\% restante (AUNAP, 2013).

El crecimiento y la intensificación de la piscicultura contribuyen al enriquecimiento de nutrientes en los ecosistemas acuáticos, principalmente por el aporte de nitrógeno y fósforo proveniente del alimento comercial suministrado, que desencadenan el incremento de la productividad primaria y mayor concentración de materia orgánica. Dependiendo de la concentración de nutrientes de menor a mayor escala, el medio podrá ser oligotrófico, mesotrófico o eutrófico (Esteves, 2011). La alteración del estado trófico conlleva a la eutrofización y es uno de los efectos de la acuicultura (FAO, 2006) al medio ambiente y una debilidad del sector, identificada nacionalmente en el análisis de debilidades, fortalezas, oportunidades y retos (AUNAP, 2013).

Cuando la piscicultura se desarrolla en sistemas artificiales, las descargas pueden ser tratadas antes de llegar al efluente, minimizando así el proceso de alteración; sin embargo, cuando el cultivo es realizado directamente en el ambiente natural, en jaulas por ejemplo, el sistema y el área de influencia quedan más expuestos al enriquecimiento de nutrientes y acumulación de materia orgánica, así las características químicas, físicas y biológicas pueden ser afectadas rápidamente dependiendo de la intensificación del cultivo, la especie cultivada (Echaniz y Vignatti, 2009) y la profundidad del cuerpo de agua utilizado (Boyd y Gautier, 2000).

El estado trófico puede ser determinado a partir de indicadores de calidad de agua (transparencia, clorofila, fósforo total y ortofosfato, principalmente) y a través de un índice, para verificar el estado del sistema. Uno de los índices más utilizados en los sistemas de cultivo, es el de Carlson (1977) y su adaptación (Carlson y Simpson, 1996) reconocidos como herramienta válida para la gestión de la calidad de agua en estos ambientes.

De acuerdo con SEAP y FAO (2008) los impactos positivos de la acuicultura son mayores frente a los negativos, tal vez por responder a la necesidad alimentaria de los pueblos y por esta misma razón el cultivo en jaulas se viene consolidando en diversos países, como una alternativa productiva, aunque propenden a la ocupación de represas y cuerpos lénticos desamparados por la política ambiental. El sistema de cultivo en jaulas de especies nativas en ambientes naturales es el sistema de mayor desarrollo en el Brasil porque permite optimizar el uso de los recursos e incrementar la capacidad de carga, además de ser un multiplicador de la producción piscícola, generador de autoempleo y fortalecimiento del núcleo familiar (Valenti, 2016).

En Colombia el ejemplo más representativo de cultivo en jaulas se desarrolla en la represa de Betania en el Huila, instaurado con soporte legal para explotación; en términos productivos responde por el $32 \%$ de la piscicultura nacional, sin embargo, ambientalmente el sistema ha demostrado síntomas de saturación, manifestado con elevadas mortalidades de tilapia, principalmente durante la época de verano, tal vez como consecuencia al haber sobrepasado la capacidad de carga establecida por el ente gubernamental en 2000 toneladas (AUNAP, 2013). Aun así, no se conocen estudios limnológicos relevantes que permitan tomar medidas al respecto.

En la región de la Orinoquía colombiana, el cultivo en jaulas también se está implementando, más aún, porque se recibe apoyo institucional y se viabilizan como emprendimientos de asociaciones en pro de la seguridad alimentaria y del mejoramiento de la calidad de vida, mediante el fortalecimiento social reflejado en el trabajo comunitario, opción laboral para pescadores de la tercera edad y contribución a la economía regional, como lo reportado por Murillo-Pacheco (2016). 
El objetivo de este trabajo fue caracterizar el área de influencia del cultivo en jaulas sobre la calidad del agua y sedimento en la ensenada-soplaviento, del río Manacacias.

\section{Materiales y métodos}

En el río Manacacias-Meta, en el punto conocido como ensenada soplaviento ( $4^{\circ} 16^{\prime} 43^{\prime \prime} \mathrm{N}$ y $\left.72^{\circ} 02^{\prime} 47^{\prime \prime} \mathrm{W}\right)$, con el aval y apoyo de entidades gubernamentales y privadas se instauró el programa: Establecimiento de un sistema de producción de cachama en jaulas flotantes como fortalecimiento a ASOPESGA, de la asociación de pescadores artesanales de Puerto Gaitán. En este contexto, se programó establecer escalonadamente la producción intensiva de cachama en nueve jaulas bajo las siguientes premisas: Cada jaula de $10.2 \mathrm{~m}^{3}$ $(3 \times 2 \times 1.7 \mathrm{~m}) 100$ peces por $\mathrm{m}^{3}$; suministro de alimento comercial extrusado con $34 \%$ de proteína, dos veces al día hasta aparente saciedad.

En estos términos, el trabajo se desarrolló el río Manacacias, acompañando la implementación (muestreo inicial=M0) y desarrollo del proyecto productivo cubriendo las etapas de: siembra (M1), levante (M2) engorda (M3) y cosecha (M4), con intervalos de 45 días entre muestreos.

Inicialmente, en agosto de 2015 fueron localizados aleatoriamente cinco puntos, cubriendo el área donde sería implementado el cultivo, así al medio día y por triplicado en cada punto, se llevó a cabo el M0 y se registraron in situ oxígeno y temperatura (YSI DO200 ${ }^{\circledR}$ ), profundidad y transparencia con disco de Secchi. Muestras de agua $(350 \mathrm{ml})$ fueron recolectadas a 30 $\mathrm{cm}$ de profundidad y seguidamente, se recolectaron muestras de sedimento con un tubo recolector tipo barreno. Las muestras de agua y sedimento fueron refrigeradas y transportadas al laboratorio de dinámica de nutrientes, en el Instituto de Acuicultura de la Universidad de los Llanos. El agua se mantuvo refrigerada hasta la realización de los análisis; el sedimento fue secado en estufa a $60^{\circ} \mathrm{C}$ durante 72 horas, macerado hasta polvo fino y almacenado en un desecador hasta la realización de los análisis.

Una vez implementado el cultivo se determinaron tres

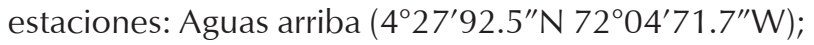
Producción $\left(4^{\circ} 27^{\prime} 96.6^{\prime \prime} \mathrm{N} 72^{\circ} 04^{\prime} 65.1^{\prime \prime} \mathrm{W}\right) ;$ y Aguas abajo $\left(4^{\circ} 28^{\prime} 01.4^{\prime \prime} \mathrm{N} 72^{\circ} 04^{\prime} 54.8^{\prime \prime} \mathrm{W}\right)$, en cada estación y por triplicado (margen, intermedio y centro del rio) se llevaron a cabo los muestreos M1,M2,M3 y M4, con el mismo procedimiento descrito para el muestreo inicial.

\section{Análisis de laboratorio}

Las muestras de agua, una vez aclimatadas fueron distribuidas para: alcalinidad, dureza, amonio y ortofosfato (APHA, 2005), para titulación fue usada una bureta digital (Technotrate Kartell $($ ) de $50 \mathrm{ml}$, para las lecturas colorimétricas un Fotómetro (YSI 930 ${ }^{\circledR}$ ); y para $\mathrm{pH}$, conductividad y sólidos totales el equipo ExTill II ${ }^{\circledR}$. En el sedimento fue determinado $\mathrm{pH}$; y materia orgánica por gravimetría.

A partir de las concentraciones de amonio y ortofosfato fueron estimados el fósforo total y el nitrógeno (EPA, 2012) para determinar el grado de trófia en las estaciones de muestreo mediante los Índices del Estado Trófico (IET) propuestos por Kratzer y Brezonik (1981) para nitrógeno y Carlson y Simpson (1996) para fósforo total.

\section{Análisis de los resultados}

La caracterización inicial del sistema (M0) en términos de calidad de agua y sedimento se realizó con estadística descriptiva (media \pm desvío estándar). Los datos de cada una de las estaciones de muestreo fueron analizados mediante la metodología de modelos mixtos, incluyendo como efectos fijos la estación de muestreo (aguas arriba, producción y aguas abajo), el muestreo (M1, M2, M3 y M4) y su interacción. Como efecto aleatorio fue considerado el sitio de muestreo (margen, intermedio y centro) dentro de la estación de muestreo. Gráficos de residuos (residuos contra ajustados e histograma) permitieron evaluar los supuestos de homocedasticidad y normalidad. Para las variables: amonio, ortofosfato, conductividad y sólidos, los valores fueron inferiores al límite de detección, en estos casos fue imputado el valor $1 / 2$ del límite de detección (Quinn y Keuugh, 2002). La transformación logaritmo natural fue aplicada a las variables, oxígeno disuelto, alcalinidad, dureza, ortofosfato, amonio, conductividad y sólidos totales para superar problemas de heterocedasticidad. La separación de medias fue efectuada mediante la prueba de Tukey. El nivel de significancia adoptado fue de $5 \%$. Los análisis fueron realizados mediante el procedimiento MIXED del programa SAS.

La clasificación trófica del sistema de cultivo fue comparada con el IET tabulado así: un valor del IET inferior a 30 representa condiciones ultraoligotróficas; entre 30 y 40, oligotróficas; 40 y 50, mesotróficas; 50 y 70 eutróficas y por encima de 70 hipereutróficas (Carlson \& Simpson, 1996).

Parámetros limnológicos del área de influencia del cultivo de cachama (Piaractus brachypomus) en jaulas. 


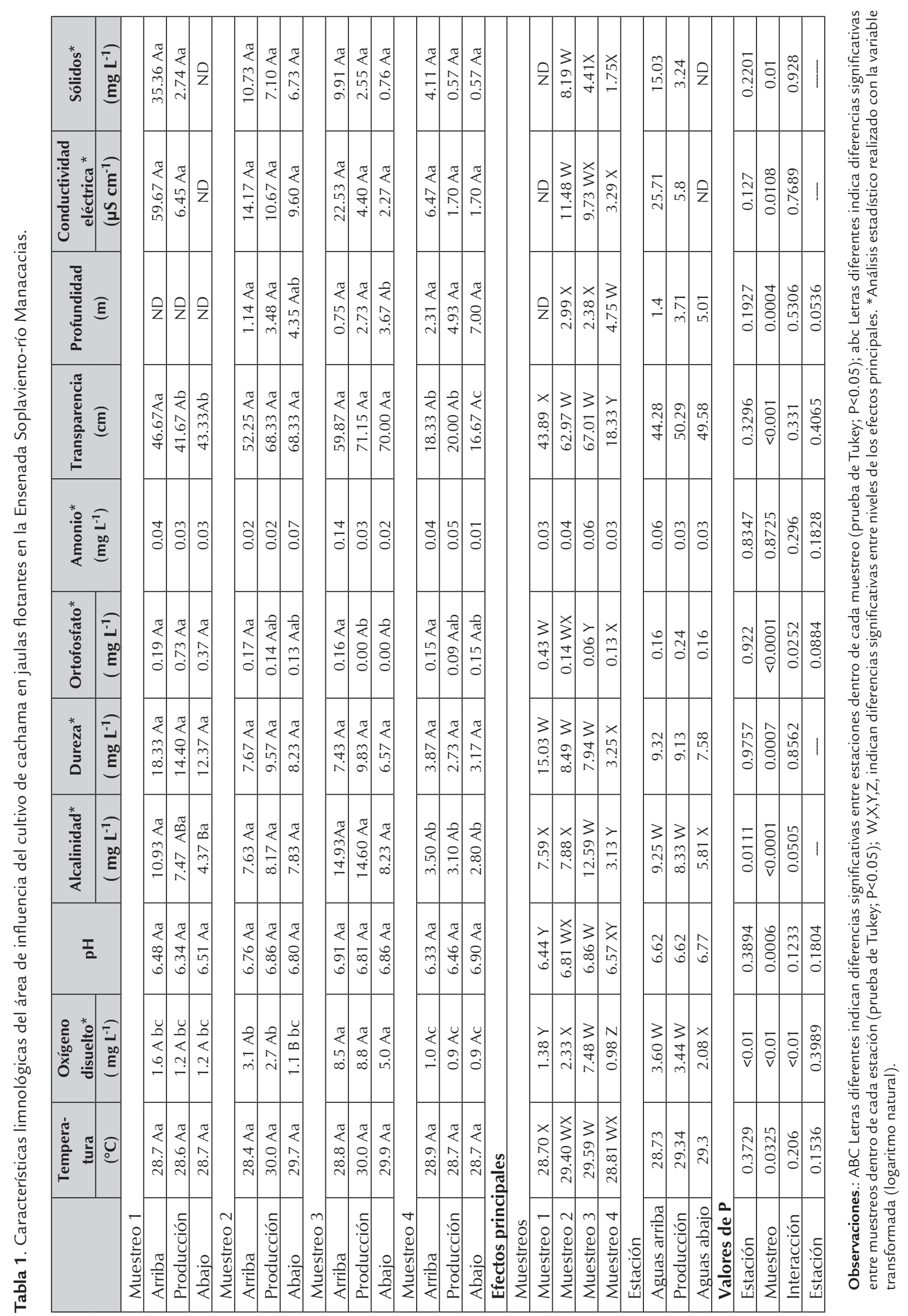


Tabla 2. Características del sedimento del área de influencia del cultivo de cachama en jaulas flotantes en la Ensenada Soplaviento, río Manacacias.

\begin{tabular}{|c|c|c|}
\hline ítem & $\begin{array}{c}\text { Materia orgánica } \\
(\%)\end{array}$ & $\mathrm{pH}$ \\
\hline \multicolumn{3}{|l|}{ Muestreo 1} \\
\hline Aguas arriba & $1.03 \mathrm{Ba}$ & 4.47 Aab \\
\hline Producción & $3.24 \mathrm{Aa}$ & $4.40 \mathrm{Aa}$ \\
\hline Aguas abajo & $2.86 \mathrm{Aa}$ & $4.30 \mathrm{Ab}$ \\
\hline \multicolumn{3}{|l|}{ Muestreo 2} \\
\hline Aguas arriba & $0.58 \mathrm{Ba}$ & $4.30 \mathrm{Ab}$ \\
\hline Producción & $3.25 \mathrm{Aa}$ & $4.85 \mathrm{Aa}$ \\
\hline Aguas abajo & $3.06 \mathrm{Aa}$ & 4.50 Aab \\
\hline \multicolumn{3}{|l|}{ Muestreo 3} \\
\hline Aguas arriba & $0.48 \mathrm{Ba}$ & $4.93 \mathrm{Aa}$ \\
\hline Producción & $3.19 \mathrm{Aa}$ & $4.80 \mathrm{Aa}$ \\
\hline Aguas abajo & $3.23 \mathrm{Aa}$ & 4.57 Aab \\
\hline \multicolumn{3}{|l|}{ Muestreo 4} \\
\hline Aguas arriba & $1.36 \mathrm{Ba}$ & $4.94 \mathrm{Aa}$ \\
\hline Producción & $4.07 \mathrm{Aa}$ & $4.70 \mathrm{Aa}$ \\
\hline Aguas abajo & $3.45 \mathrm{Aa}$ & $5.11 \mathrm{Aa}$ \\
\hline \multicolumn{3}{|c|}{ Efectos Principales } \\
\hline \multicolumn{3}{|l|}{ Muestreos } \\
\hline Muestreo 1 & 2.38 & $4.39 \mathrm{Y}$ \\
\hline Muestreo 2 & 2.3 & $4.55 X Y$ \\
\hline Muestreo 3 & 2.3 & $4.77 W X$ \\
\hline Muestreo 4 & 3 & $4.92 \mathrm{~W}$ \\
\hline \multicolumn{3}{|l|}{ Local } \\
\hline Aguas arriba & $0.86 X$ & 4.66 \\
\hline Producción & $3.44 \mathrm{~W}$ & 4.69 \\
\hline Aguas abajo & $3.15 \mathrm{~W}$ & 4.62 \\
\hline \multicolumn{3}{|l|}{ Valores de P } \\
\hline Local & $<0.0001$ & 0.8939 \\
\hline Muestreo & 0.0707 & 0.0005 \\
\hline Interacción & 0.6746 & 0.015 \\
\hline Estación & - & 0.1316 \\
\hline
\end{tabular}

Observaciones.: ABC Letras diferentes indican diferencias significativas entre estaciones dentro de cada muestreo (prueba de Tukey; $P<0.05$ ); abc Letras diferentes indica diferencias significativas entre muestreos dentro de cada estación (prueba de Tukey; $\mathrm{P}<0.05)$; WXYZ indican diferencias significativas entre niveles de los efectos principales (prueba de Tukey; $\mathrm{P}<0.05$ ).

\section{Resultados}

Con el muestreo inicial (M0) se caracterizó el área donde se implementó el emprendimiento productivo (M0) así: temperatura $26^{\circ} \mathrm{C}( \pm 0.05)$, oxígeno disuelto 4.63 mg. $\mathrm{L}^{-1}( \pm 0.08)$, profundidad $5.3 \mathrm{~m}( \pm 0.11), \mathrm{pH} 6.61$ $( \pm 0.35)$, alcalinidad $0.34 \mathrm{mg}^{-\mathrm{L}^{-1}}( \pm 0.26)$, dureza 11.65 mg.. ${ }^{-1}( \pm 11.82)$, ortofosfato $0.34{\mathrm{mg} . \mathrm{L}^{-1}}^{-1} \pm 0.26)$, amonio $0.14 \mathrm{mg} \cdot \mathrm{L}^{-1}( \pm 0.14)$, transparencia $27 \mathrm{~cm}( \pm 2.74)$, conductividad $8.24 \mu \mathrm{S} \mathrm{cm} \mathrm{cm}^{-1}( \pm 1.51)$ y sólidos totales 5.28 mg. $\mathrm{L}^{-1}( \pm 1.91)$, en agua; en sedimento, $\mathrm{pH} 5.46$ $( \pm 0.04)$ y materia orgánica $3.6 \%( \pm 0.10)$.

Los resultados de los parámetros analizados en los muestreos correspondientes al desarrollo del proyecto se registran en la tabla 1 para agua y para sedimento en la tabla 2. Referente al estado trófico en la estación producción se denotaron durante el proceso condiciones ultraoligotróficas $(M 1=16)$, mesotróficas $(M 2-M 3<50)$ e hipereutróficas $(M 3=84)$ en términos de IET para fósforo total, en cuanto que para nitrógeno, durante todo el proceso fueron ultraoligotróficas $(\mathrm{IET}<6)$.

\section{Discusión}

El río Manacacias corre de sur a norte en un recorrido aproximado de $87 \mathrm{~km}$ y desemboca en el río Meta. Se clasifica como un río de aguas blancas (Sioli, 1984) ya que esta tipología de aguas nacen en los Andes que contribuye con sedimentos a consecuencia de erosiones, dando el color café con leche o amarillo característico, otro aspecto propio de estas aguas es un $\mathrm{pH}$ cercano a neutro y transparencia inferior a $50 \mathrm{~cm}$ debido al alto contenido de arcillas. En el muestreo inicial en el río Manacacias, los valores de conductividad, dureza alcalinidad y sólidos fueron respectivamente $8.24 \mu \mathrm{s}$, $5.82 \mathrm{mg} . \mathrm{L}^{-1}, 3.88 \mathrm{mg} . \mathrm{L}^{-1}$ y $5.28 \mathrm{mg} . \mathrm{L}^{-1}$, correspondiendo a valores incrementados en $100 \%$ a los reportados por Geoingenieria en el 2010, entre tanto, ortofosfato (0.35 mg. $\left.\mathrm{L}^{-1} \mathrm{l}\right)$ y amonio $\left(0.14 \mathrm{mg}^{-\mathrm{L}^{-1}}\right)$ no habían sido registrados. Los valores medios para oxígeno disponible (4.63 mg. $\left.\mathrm{L}^{-1}\right), \mathrm{pH}(6.61)$ y temperatura $\left(26.76{ }^{\circ} \mathrm{C}\right)$ corresponden con valores característicos en ríos de aguas blancas, como el Amazonas, el Orinoco y el Uyucaly, hábitats de la cachama, de acuerdo a la recopilación de Araújo-Lima y Goulding (1998).

Durante el seguimiento al sistema de cultivo, para siete de la once variables analizadas (Tabla 1 ) fue encontrado efecto significativo del muestreo $(P<0.05), y$ se puede interpretar que el muestreo asociado a los ciclos de lluvia influenciaron de manera acentuada los parámetros de calidad de agua. Para oxígeno disuelto y alcalinidad la interacción fue significativa $(P<0.01$ y 
$\mathrm{P}=0.05$ respectivamente), indicando que los cambios generados por el efecto de la producción y/o local de muestreo, son también dependientes del muestreo, por ejemplo, una disminución en el nivel de oxígeno disuelto fue evidenciado solo en el muestreo 2, el efecto sobre la alcalinidad solo fue encontrado en el muestreo 1. Con todo, las variables de calidad de agua se mantuvieron en el rango considerado adecuado para el cultivo de la cachama, este aspecto es una de las ventajas de cultivar especies nativas en ambiente natural, como reportado para Colossoma macropomum por Frias et al., (2011) en el rio Naney en Perú.

En términos generales en el área de producción, el valor medio de $\mathrm{pH}(6.63 \pm 0.24)$ pudo haber contribuido con la estabilidad del sistema, restringiendo la formación de amonio (0.03 mg. $\left.\mathrm{L}^{-1}\right)$, cuyos niveles no fueron afectados por ninguno de los factores estudiados, propiciando así un ambiente adecuado para los peces; resultado similar fue reportado por Gorlach-Lira et al., 2013 en cultivo de tilapia en el lago Padre Azevedo en Brasil.

La materia orgánica del sedimento fue afectada significativamente por la estación de muestreo, siendo que en aguas arriba se presentó niveles más bajos de materia orgánica $(0.48 \%)$, se puede sugerir que el principal impacto de la producción es un acúmulo de materia orgánica tanto en la producción (3.44\%) como también aguas abajo (3.15\%), por otro lado, esta concentración puede ser el resultado del efecto de la corriente que desplaza los nutrientes rio abajo. En el lago Guamuez en Nariño, la concentración de materia orgánica en el cultivo de truchas estaría aportando un incremento de $46 \%$ en materia orgánica en relación con el punto de control (González, 2017); la concentración de materia orgánica es otro punto antagónico al optar por cultivos en sistemas naturales.

En el área de producción el estado de trófia en términos de fósforo vario entre mesotrófico e hipereutrófico coincidiendo con meses de mayor retención hídrica debido a época de escasa precipitación (M3=mes de febrero), y menor profundidad $(2.75 \mathrm{~m})$, como lo registrado por Bonh et al., (2012) en lagunas Pampanas donde relacionaron el efecto de la pluviosidad sobre la concentración de nutrientes.

Considerando la relevancia del fósforo y nitrógeno como indicadores de enriquecimiento, se denota que la dilución de nutrientes en la ensenada soplaviento estaría a cargo de la propia dinámica de reciclaje del sistema, como recopilado por Américo, et al., (2013) en cultivos en jaulas en represas brasileras, además, en sistemas lénticos el aporte de fósforo (9\%) y nitróge- no $(25 \%)$ del cultivo de tilapia en jaulas representa un pequeño porcentual en relación con la carga anual del sistema (Lacerda et al., 2018).

Teniendo en cuenta que durante el ciclo productivo estudiado, no fueron detectadas alteraciones drásticas, entre los aspectos que pueden estar contribuyendo a la disminución de efectos atenuantes (bajas concentraciones de fósforo, nitrógeno y materia orgánica), estarían: la especie en cultivo, que al ser nativa responde óptimamente a las características del medio; la biomasa producida (4 toneladas por ciclo) no estaría comprometiendo la capacidad de carga del sistema y las prácticas de manejo llevadas a cabo por los integrantes de ASOPESGA controlarían los aportes de nutrientes, además, de acuerdo con la recopilación hecha por Carvalho y Paiva (2010) en hidroeléctricas brasileras, es necesario como mínimo cinco años de funcionamiento y monitoreo en los cultivos en jaulas para detectar cambios en el sistema.

\section{Conclusiones}

De acuerdo a los resultados, en la ensenada soplaviento aún no se está reflejando alguna alteración en el estado trófico por el sistema de cultivo allí implementado, estos resultados contribuyen a la estructuración de la línea base limnológica del rio Manacacias y podrán ser cruzados con las informaciones económicas y sociales del proyecto para estudiar la sostenibilidad del emprendimiento asociativo.

\section{Agradecimientos}

A la dirección general de investigaciones-DGl de Unillanos por el financiamiento a través de la convocatoria 01 IALL-01-14; a la asociación de pescadores artesanales de Puerto Gaitán (ASOPESGA), especialmente a Leyton; al profesor Ricardo Murillo por la articulación sector productivo-academia y a los integrantes del grupo Granac por su apoyo en el trabajo de campo.

\section{Referencias}

Américo JHP, Torres NH, Machado AA, Carvalho SL. 2013. Piscicultura em tanques-rede: impactos e consequências na qualidade da água. Revista Científica ANAP Brasil, 6 (7):137-150. APHA. 2005. Standard methods: for the examination of water and wastewater. 21a ed. American Public Health Association. EUA. 1368 p.

Araújo-Lima C, Goulding M. 1998. Os frutos do tambaqui: ecologia, conservação e cultivo na Amazônia. Brasília: MCT-CNPq, 186p.

AUNAP. 2013. Diagnostico del estado de la acuicultura en Colombia. Ministerio de Agricultura y Desarrollo Rural, Colombia 160 p. 
Bohn VY, Piccolo MC, Perillo GM, Pratolongo PD, Fornerón CF. Caracterización óptica de las lagunas Sauce Grande y Chasicó (provincia de Buenos Aires). Biol Acuat. 2012;(27):51-60.

Boyd CE, Gautier D. Effluent composition and water quality standards. Global Aquaculture Advocate. 2000;3(5):61-66.

Carlson RE, Simpson J. 1996. A coordinator's guide to volunteer lake monitoring methods. North American Lake Management Society, Madison.

Carlson RE. A trophic state index for lakes. Limnol Oceanogr. 1977;22(2):361-369.

Carvalho ED, Paiva RI. Aqüicultura Em Grandes Represas Brasileiras: Interfaces Ambientais, Socioeconômicas E Sustentabilidade. Boletim da Sociedade Brasileira de Limnologia. 2010;38(1):118.

Echaniz S, Vignatti A. determinación del estado trófico y de la capacidad de carga del embalse casa de piedra. BioScriba. 2009;2(1):41-51.

EPA, 2012. Integrated Water Quality Report Monaghan y Louth 2011. EPA, Wexford.

Esteves FDA. 2011. Fundamentos de limnologia. 3ra. Edição Interciência/Finep.

FAO 2006. El estado mundial de la pesca y la acuicultura. Roma. Organización de las Naciones Unidas para la Agricultura y la Alimentación, 178p.

FAO 2018. El estado mundial de la pesca y la acuicultura. Roma. Organización de las Naciones Unidas para la Agricultura y la Alimentación, 210p.

Frias AR, Tecco JS, Díaz RP, Acosta NYB, Herrera ERT, Vela CMS, Cubas GR, et al. Influencia de la densidad en policultivo de Colossoma macropomum y Prochilodus nigricans, en jaulas flotantes. Río Nanay, Loreto, Perú. Conocimiento Amazónico. 2015;2(1):3-12.

Geoingenieria. 2010. Estudio del impacto ambiental línea eléctrica de 230 Kv subestación Chivor-Campo Rubiales, capitulo 4.0. Informe técnico.180 p.

González LEA. 2017. Impacto ambiental de la acuicultura intensiva en los componentes agua y sedimento en el lago Guamuez, Nariño. Maestría thesis, Universidad Nacional de Colombia - Sede Palmira.

Gorlach-Lira K, Pacheco C, Carvalho LCT, Melo Júnior HN, Crispim MC. The influence of fish culture in floating net cages on microbial indicators of water quality. Braz J Biol. 2013;73(3):457-463.

Kratzer CR, Brezonik PL. A Carlson-type trophic state index for nitrogen in Florida lakes. Water Res Bull. 1981;17:713-715.

Lacerda LD, Santos JA, Marins RV, Silva FAD. Limnology of the largest multi-use artificial reservoir in NE Brazil: The Castanhão Reservoir, Ceará State. An. Acad Bras Ciênc. 2018;90(2):20732096.

Murillo-Pacheco R. 2016. Experiencias de cultivo en jaulas flotantes con especies ícticas nativas en el departamento del Meta, Colombia. XXII Jornada de acuicultura IALL. 52-58.

Quinn G, Keough M. Experimental design and data analysis for biologist. Cambrige University press.

SEAP Secretaria Especial de Aqüicultura e Pesca y FAO Organização das Nações Unidas para Agricultura e Alimentação. 2008. Aqüicultura no Brasil: o desafio é crescer. Brasília, Brazil.

Sioli H. 1984. The Amazon and its main affluents: hydrography, morphology of the river courses, and river types. The Amazon. Pp.127-165. Springer Netherlands. 